\title{
Analysis of the TeV-scale mirage mediation with heavy superparticles
}

\author{
Junichiro Kawamura $^{a}$ and Yuji Omura ${ }^{b}$ \\ ${ }^{a}$ Department of Physics, University of Tokyo, \\ Tokyo 113-0033, Japan \\ ${ }^{b}$ Kobayashi-Maskawa Institute for the Origin of Particles and the Universe (KMI), \\ Nagoya University, Nagoya 464-8602, Japan \\ E-mail: kawamura@hep-th.phys.s.u-tokyo.ac.jp, \\ yujiomur@kmi.nagoya-u.ac.jp
}

ABSTRACT: We discuss effective models derived from a supersymmetric model whose mediation mechanism of supersymmetry (SUSY) breaking is namely mirage mediation. In this model, light higgsino mass, that is required by the natural realization of the electroweak scale, is achieved by the unification of the soft SUSY breaking parameters at the low scale. Besides, we find that extra Higgs fields are also possibly light in some cases. Then, the effective model is a two Higgs doublet model (2HDM) with higgsinos, and it is distinguishable with namely type-II $2 \mathrm{HDM}$ which is widely discussed. In this paper, we study the mass spectrum of SUSY particles and the extra Higgs fields, and summarize the phenomenology in the effective model. We survey the current experimental bounds from the LHC and the dark matter experiments as well as the flavor physics. Then, we point out the expected mass scale of the SUSY particles and reveal the future prospects for the direct and indirect searches. We also discuss the difference between our effective model and the 2HDM in the bottom-up approach.

KeYWORDS: Supersymmetry Phenomenology

ARXIV EPRINT: 1710.03412 


\section{Contents}

1 Introduction 1

2 Mirage mediation $\quad 2$

3 Phenomenology 4

3.1 Electroweak symmetry breaking $\quad 6$

3.2 Direct LHC search 8

3.3 Precision Higgs coupling measurement 9

$\begin{array}{llr}3.4 & \text { Flavor physics } & 10\end{array}$

3.5 DM physics 11

4 Numerical analysis $\quad 13$

5 Conclusion $\quad 16$

\section{Introduction}

One elegant explanation for the origin of the electroweak (EW) scale is given by the supersymmetric extension of the Standard Model (SM). ${ }^{1}$ In the Minimal Supersymmetric Standard Model (MSSM), the superpartners of the SM particles are introduced and quadratic divergence is canceled out in the Higgs mass squared. The natural realization of the EW scale predicts the superpartners to be the EW-scale, so that a lot of efforts have been devoted to discover the new particles. The latest LHC results on the supersymmetry (SUSY) search, however, show that SUSY particles do not exist below a few TeV in a simple scenario. In addition, the Higgs discovery around the $125 \mathrm{GeV}$ mass [3] indirectly constraints the SUSY scale, since the MSSM predicts that the mass of a neutral Higgs particle is much lower than $125 \mathrm{GeV}$ without large radiative corrections. Then, we might conclude that the SUSY scale is much higher than the EW scale.

On the other hand, it is true that the relation between the SUSY scale and the realization of the EW scale is not so simple in the MSSM. In fact, we can find some explicit SUSY models that are able to be consistent with the experimental results and explain the origin of the EW scale naturally. One simple way is to consider the MSSM with non-universal gaugino masses at the unification scale $\left(\sim 10^{16} \mathrm{GeV}\right)[4,5]$. A specific mass ratio of wino to gluino can realize the EW scale naturally, even if gluino is heavy. Then, we can evade the strong bounds from the direct SUSY search and explain the $125 \mathrm{GeV}$ Higgs mass [6, 7]. The mass ratio is non-trivial, but it is known that such a unique mass spectrum is predicted by a mediation mechanism: namely mirage mediation [8-10]. In this mechanism, the moduli mediation [11-13] and the anomaly mediation [14, 15] are compatible, and the

\footnotetext{
${ }^{1}$ See for a reviews, e.g. $[1,2]$
} 
renormalization-group ( $\mathrm{RG}$ ) correction of the moduli-mediation contribution is canceled by the anomaly-mediation. Phenomenology of the mirage mediation have been studied before the Higgs boson discovery [16-18] and after the discovery [19-23]. The mirage mediation in the Next-to-MSSM ${ }^{2}$ has been also studied in refs. [19, 20, 22].

In the last few years, the LHC run-II excludes light SUSY particles (sparticles); especially, colored sparticles, namely squarks and gluinos, have to be heavier than $2 \mathrm{TeV}$. Therefore it is worth to study the scenarios that heavy colored sparticles can be compatible with the natural explanation of the EW scale.

In this paper, we reconsider the mirage mediation and discuss the phenomenology based on the latest experimental results. Even if the sparticles, except for the superpartner of the Higgs field (higgsino), are much heavier than the LHC reach, the extra Higgs bosons and the higgsino become lighter than $\mathrm{TeV}$ scale in our scenario. The light particles can be tested by the direct LHC search, flavor experiments, precision measurements of the Higgs boson couplings and dark matter (DM) searches. Based on the integrated research, we point out the expected mass scale of the SUSY particles and reveal the future prospects for the direct and indirect searches. We also discuss the difference between our effective model and the 2HDM in the bottom-up approach.

This paper is organized as follows. The mirage mediation is briefly reviewed in section 2, and phenomenology of the mirage mediation is discussed in section 3 . The results of numerical analysis are shown in section 4 . Section 5 is devoted to conclusion.

\section{Mirage mediation}

The mirage mediation is a mixture of the modulus and anomaly mediations. Once the mediation mechanism of the supersymmetry breaking is specified, the soft SUSY breaking terms, given by

$$
-\mathcal{L}_{\mathrm{soft}}=\frac{1}{2} M_{a} \lambda_{a} \lambda_{a}+\frac{1}{2} m_{i}^{2}\left|\phi^{i}\right|^{2}+\frac{1}{6} A_{i j k} y_{i j k} \phi^{i} \phi^{j} \phi^{k}+\text { h.c. , }
$$

are determined. $\lambda_{a}$ and $\phi^{i}$ are gauginos in vector supermultiplets and scalar fields in chiral supermultiplets. In our notation, we factorize scalar trilinear couplings as $A_{i j k} y_{i j k}$, where $y_{i j k}$ is a Yukawa coupling. The indices $a$ and $i$ run over the MSSM gauge groups and the scalar fields, respectively.

In the mirage mediation, the soft parameters at the unification scale $M_{U}$ are given by

$$
\begin{aligned}
M_{a}\left(M_{U}\right)= & M_{0}\left[1+\frac{b_{a}}{16 \pi^{2}} g_{0}^{2} \alpha \ln \frac{M_{p}}{m_{3 / 2}}\right], \\
A_{i j k}\left(M_{U}\right)= & -M_{0} \sum_{l=i, j, k}\left[c_{l}-\frac{\gamma_{l}}{16 \pi^{2}} \alpha \ln \frac{M_{p}}{m_{3 / 2}}\right], \\
m_{i}^{2}\left(M_{U}\right)= & M_{0}^{2}\left[c_{i}-\frac{1}{32 \pi^{2}} \frac{d \gamma_{i}}{d \ln Q}\left(\alpha \ln \frac{M_{p}}{m_{3 / 2}}\right)^{2}\right. \\
& \left.+\frac{1}{4 \pi^{2}}\left(\frac{1}{4} \sum_{j, k}\left|y_{i j k}\right|^{2} \sum_{l=i, j, k} c_{l}-\sum_{a} g_{a}^{2} C_{2}^{a}\left(\phi^{i}\right)\right) \alpha \ln \frac{M_{p}}{m_{3 / 2}}\right],
\end{aligned}
$$

${ }^{2}$ See for a review, e.g. [24]. 
The first and second terms in these expressions correspond to the modulus and the anomaly mediation, respectively. The last term in eq. (2.4) comes from both of the mediation mechanisms. We parametrize the overall size of modulus mediation by $M_{0}$ and each $c_{i}$ describes the ratio of the scalar mass parameter of $\phi_{i}$ to $M_{0}$. The size of $c_{i}$ depends on the coupling of $\phi_{i}$ with the modulus. Note that $c_{i}$ is discrete in the string models. $g_{0}$ is the unified gauge coupling at $M_{U}$ and $C_{2}^{a}\left(\phi^{i}\right)$ is the quadratic Casimir for a scalar $\phi^{i}$. $b_{a}=(33 / 5,1,-3)$ is the beta-function coefficients for the MSSM gauge couplings. $\gamma_{i}$ is an anomalous dimension for a scalar $\phi^{i}$ and given by

$$
\gamma_{i}=2 \sum_{a} g_{a}^{2} C_{2}^{a}\left(\phi^{i}\right)-\frac{1}{2} \sum_{j, k}\left|y_{i j k}\right|^{2} .
$$

$d \gamma_{i} / d \ln Q$ is a derivative of $\gamma_{i}$ with respect to the logarithmic of the renormalization scale $Q$.

We parametrize the ratio of the anomaly mediation to the modulus mediation by $\alpha$ and it is defined as

$$
\alpha \equiv \frac{m_{3 / 2}}{M_{0} \ln \left(M_{p} / m_{3 / 2}\right)}
$$

where $M_{p} \simeq 2.4 \times 10^{18} \mathrm{GeV}$ is the Planck mass and $m_{3 / 2}$ is the gravitino mass. This parametrization is motivated by the KKLT-type moduli stabilization scenario [25-27]. Note that $\ln \left(M_{p} / m_{3 / 2}\right) \sim \mathcal{O}\left(8 \pi^{2}\right)$ and compensate the loop suppression factors appeared in the anomaly mediated contributions. $\alpha=1$ is realized in the original KKLT-setup [28], but various rational values can be obtained in the similar setups [8, 9, 29, 30]. An important fact is that $\alpha$ could be determined by rational parameters, such as the winding number of D-branes and the number of fluxes which generates moduli potential.

A remarkable feature of the mirage mediation is the unification of some parameters at the low scale (mirage unification) [8-10]. Assuming that the moduli-mediation contributions satisfy

$$
c_{i}+c_{j}+c_{k}=1
$$

the anomaly-mediation contributions cancel out the RG corrections of the modulusmediation contributions at a scale $\left(M_{\text {mir }}\right)$. The unification scale, namely mirage scale, is given by

$$
M_{\text {mir }}=\frac{M_{U}}{\left(M_{p} / m_{3 / 2}\right)^{\alpha / 2}},
$$

and the soft parameters at $M_{\text {mir }}$ are estimated as

$$
M_{a}\left(M_{\mathrm{mir}}\right)=M_{0}, A_{i j k}\left(M_{\mathrm{mir}}\right)=-M_{0}, m_{i}^{2}\left(M_{\mathrm{mir}}\right)=c_{i} M_{0}^{2} .
$$

If $\alpha \sim 2$, the mirage scale is around TeV-scale. In our analysis, we assume $M_{\text {mir }}=M_{\text {SUSY }} \equiv$ $\sqrt{m_{Q_{3}} m_{u_{3}}}$, where $m_{Q_{3}}, m_{u_{3}}$ are soft masses for left-handed and right-handed top squarks, respectively.

Equation (2.9) shows that $m_{H_{u}}^{2}$ at the mirage scale vanishes at the leading order if $c_{H_{u}}=0$ is satisfied. This means that $|\mu|^{2} \simeq m_{H_{u}}^{2}$ is EW-scale even when the other scalar 
masses, which are estimated as $\mathcal{O}\left(M_{0}\right)$, are much larger than the EW scale. If the mirage condition is satisfied and the A-term is not larger than the scalar masses for the top squarks, $M_{0}$ need to be larger than about $7 \mathrm{TeV}$ in order to explain the SM-like Higgs boson mass as discussed later. Thus, the $125 \mathrm{GeV}$ Higgs boson mass and heavy sparticles are achieved, while the EW scale is realized without fine-tunings in the TeV-scale mirage mediation.

In our analysis, we assume

$$
c_{Q}=1 / 2, c_{H_{u}}=c_{H_{d}}=0,
$$

where $c_{Q}$ is for all scalar particles other than $H_{u, d}$. Note that $c_{H_{d}}=0$ has to be satisfied in order to realize the mirage unification when $\tan \beta$ is large and the bottom and tau Yukawa couplings are also sizable adding to the top Yukawa coupling.

As pointed out in refs. [8, 9], the sub-leading corrections to the mass squared become important for parameters that vanish at the tree-level. Hence, we assume that $m_{H_{u, d}}^{2}$ is small but not vanishing at the mirage scale:

$$
m_{H_{u, d}}^{2}\left(M_{\text {mir }}\right)=\delta m_{H_{u, d}}^{2}=\mathcal{O}\left(\frac{M_{0}^{2}}{8 \pi^{2}}\right) .
$$

The sub-leading correction would come from the fluctuation of the mirage scale from $M_{\mathrm{SUSY}}$, higher-loop corrections in both the MSSM and UV-models, and sub-leading corrections in moduli stabilizations. Those corrections are expected to be small, so that only the higgsino and the extra Higgs bosons are below sub-TeV, while all the other sparticles are expected to be heavier than the sub-TeV scale.

\section{Phenomenology}

In this section, we study phenomenology when the soft parameters are given by

$$
M_{a}\left(M_{\mathrm{SUSY}}\right)=-A_{i j k}\left(M_{\mathrm{SUSY}}\right)=M_{0}, m_{i}^{2}\left(M_{\mathrm{SUSY}}\right)=\frac{M_{0}^{2}}{2},
$$

and

$$
m_{H_{u, d}}^{2}\left(M_{\mathrm{SUSY}}\right)=\mathcal{O}\left(\frac{M_{0}^{2}}{8 \pi^{2}}\right) .
$$

This alignment is predicted by the $\mathrm{TeV}$-scale mirage mediation, as discussed in previous section. We have the following parameters:

$$
\tan \beta, M_{0}, m_{A}, \mu \text {. }
$$

Note that the CP-odd Higgs boson mass $m_{A}$ and the $\mu$-parameter are treated as input parameters instead of soft parameters $m_{H_{u}}^{2}$ and $m_{H_{d}}^{2}$. The size of modulus mediation $M_{0}$ is fixed to explain the SM-like Higgs boson mass.

In this setup, all of sparticles, except for higgsinos, reside far above the LHC reach, while the Higgs bosons are expected to be around $\mathrm{TeV}$-scale. One promising way is the direct search for extra Higgs bosons and the higgsino at collider experiments. Besides, the 


\begin{tabular}{|c|c|c|c|c|}
\hline Parameters & (a) & (b) & (c) & (d) \\
\hline $\tan \beta$ & 15 & 45 & 30 & 10 \\
\hline$\mu[\mathrm{GeV}]$ & -1090 & -1068 & 1000 & 100 \\
\hline$M_{0}[\mathrm{GeV}]$ & 9896 & 6783 & 7905 & 26700 \\
\hline \multicolumn{5}{|l|}{ Degree of tuning } \\
\hline$\Delta_{\mu}$ & 285.8 & 274.3 & 240.5 & 2.405 \\
\hline$\Delta_{M_{0}}$ & 524.6 & 255.0 & 341.1 & 3489 \\
\hline \multicolumn{5}{|l|}{ Mass $[\mathrm{GeV}]$} \\
\hline$m_{h}$ & 125.09 & 125.09 & 125.09 & 125.10 \\
\hline$m_{H}$ & 1477 & 1364 & 1429 & 1490 \\
\hline$m_{A}$ & 1477 & 1364 & 1429 & 1489 \\
\hline$m_{H^{ \pm}}$ & 1485 & 1362 & 1431 & 1571 \\
\hline$m_{\tilde{\chi}_{1}^{0}}$ & 1117 & 1088 & 1022 & 105.4 \\
\hline$m_{\tilde{t}_{1}}$ & 7004 & 4767 & 5578 & 18975 \\
\hline$m_{\tilde{t}_{2}}$ & 7264 & 4980 & 5811 & 19465 \\
\hline \multicolumn{5}{|l|}{ Long-Lived Particle search } \\
\hline$\Delta m_{+}[\mathrm{GeV}]$ & 0.827 & 1.071 & 0.918 & 0.395 \\
\hline$c \tau[\mathrm{mm}]$ & 0.241 & 0.0806 & 0.156 & 4.20 \\
\hline \multicolumn{5}{|l|}{ Branch/cross section } \\
\hline $\operatorname{Br}(H \rightarrow b b)$ & 0.801 & 0.872 & 0.854 & 0.609 \\
\hline $\operatorname{Br}(H \rightarrow \tau \tau)$ & 0.125 & 0.126 & 0.141 & 0.0971 \\
\hline $\operatorname{Br}\left(H^{ \pm} \rightarrow t b\right)$ & 0.866 & 0.856 & 0.860 & 0.886 \\
\hline $\operatorname{Br}\left(H^{ \pm} \rightarrow \tau \nu\right)$ & 0.131 & 0.142 & 0.138 & 0.101 \\
\hline$\sigma(p p \rightarrow H / A+b)[\mathrm{fb}]$ & 1.837 & 30.4 & 9.19 & 0.706 \\
\hline \multicolumn{5}{|l|}{ Indirect observables } \\
\hline$\kappa_{b}$ & 1.011 & 1.014 & 1.012 & 1.011 \\
\hline$\kappa_{\tau}$ & 1.011 & 1.014 & 1.012 & 1.011 \\
\hline $\operatorname{Br}(b \rightarrow s \gamma) \times 10^{4}$ & 3.39 & 3.41 & 3.40 & 3.38 \\
\hline $\operatorname{Br}\left(B_{s} \rightarrow \mu^{+} \mu^{-}\right) \times 10^{9}$ & 2.97 & 1.92 & 3.08 & 3.02 \\
\hline \multicolumn{5}{|l|}{ DM observables } \\
\hline$\Omega_{\text {thermal }} h^{2}$ & 0.119 & 0.119 & 0.102 & 0.00116 \\
\hline$\langle\sigma v\rangle_{v=0} \times 10^{25}\left[\mathrm{~cm}^{3} / \mathrm{s}\right]$ & 0.0873 & 0.0946 & 0.105 & 3.95 \\
\hline$\sigma_{\mathrm{SI}} \times 10^{11}[\mathrm{pb}]$ & 0.614 & 1.41 & 2.11 & 0.152 \\
\hline$\sigma_{\mathrm{SD}} \times 10^{8}[\mathrm{pb}]$ & 0.769 & 1.96 & 1.52 & 8.97 \\
\hline
\end{tabular}

Table 1. Values of parameters, masses, widths, branching ratios, production cross section of $H / A$ in association with a b-quark, $\kappa_{b, \tau}$, flavor observables and DM observables at benchmark points (a)-(d). 
extra Higgs bosons lead deviations from the SM predictions in some processes such as, $B_{s} \rightarrow \mu^{+} \mu^{-}, B \rightarrow X_{s} \gamma$ and the decay of the $125-\mathrm{GeV}$ Higgs boson. The higgsino becomes the lightest supersymmetric particle (LSP) in this setup. Hence the neutral component of the higgsino is a good DM candidate and the direct and indirect DM searches give significant bounds on our model.

The mass spectrum and the values of observables at several benchmark points are summarized in table 1. We calculated the mass spectrum of sparticles and their decays by using SuSpect-v2.41 [31] and SDECAY [32] interfaced by SUSY-HIT-v1.5a [33]. The Higgs boson masses, decays and couplings to the SM fermions are calculated by using FeynHiggs-v2.12.2 [34-39]. $M_{0}$ should be larger than about $6.8 \mathrm{TeV}$ in order to explain the $125 \mathrm{GeV}$ Higgs boson mass, so that superpartners except higgsino are much higher than the LHC reach.

\subsection{Electroweak symmetry breaking}

First of all, we analyze the condition for the EW symmetry breaking. In the MSSM, the Z-boson mass $m_{Z}$ is related to the parameters of the MSSM as

$$
\frac{m_{Z}^{2}}{2}=\frac{\tilde{m}_{H_{d}}^{2}-\tilde{m}_{H_{u}}^{2} \tan ^{2} \beta}{\tan \beta^{2}-1}-|\mu|^{2} \simeq-\tilde{m}_{H_{u}}^{2}-|\mu|^{2},
$$

where $\tan \beta \gg 1$ is assumed in the last equality. $\tilde{m}_{H_{u, d}}^{2}$ include corrections from the effective potential,

$$
\tilde{m}_{H_{u, d}}^{2}=m_{H_{u, d}}^{2}+\left.\frac{1}{2\left\langle H_{u, d}\right\rangle} \frac{\partial \Delta V}{\partial H_{u, d}}\right|_{H_{u, d} \rightarrow\left\langle H_{u, d}\right\rangle} \equiv m_{H_{u, d}}^{2}+\Delta m_{H_{u, d}}^{2}
$$

where $\Delta V$ is loop corrections to the effective potential.

According to the assignment of the modular weights in eq. (2.10), the $m_{H_{u}}^{2}$ at a scale $Q$ can be expressed as [27]

$$
m_{H_{u}}^{2}(Q)=\frac{M_{0}^{2}}{4 \pi^{2}}\left\{\gamma_{H_{u}}(Q)-\frac{1}{2} \frac{d \gamma_{H_{u}}(Q)}{d \ln Q} \ln \left(\frac{M_{\mathrm{mir}}}{Q}\right)\right\} \ln \left(\frac{M_{\mathrm{mir}}}{Q}\right)+\delta m_{H_{u}}^{2}(Q),
$$

where the anomalous dimension for the up-type Higgs boson is given by

$$
\gamma_{H_{u}}=\frac{3}{2} g_{2}^{2}+\frac{3}{10} g_{1}^{2}-3 y_{t}^{2} .
$$

Note that the modulus contribution to the Higgs bosons is absent at $Q=M_{\text {mir }}$ because of $c_{H_{u}}=c_{H_{d}}=0$. The RG effects through the $\mathrm{U}(1)_{Y}$ gauge coupling are also vanishing in our setup of the modulus mediation.

Let us discuss the sensitivity of the Z-boson mass with respect to the parameters in the mirage mediation [40]:

$$
\Delta=\max _{a} \Delta_{a} \equiv \max _{a}\left|\frac{d \ln m_{Z}^{2}}{d \ln a}\right|, a=|\mu|^{2}, M_{0}^{2}, \delta m_{H_{u}}^{2} .
$$


Here we do not consider tuning of $\delta m_{H_{d}}$ and the b-term because they are suppressed by $\tan ^{2} \beta$ and expected to be small $[8,9]$. The degrees of tuning of the $\mu$-parameter and $\delta m_{H_{u}}^{2}$ are given by

$$
\Delta_{\mu} \equiv\left|\frac{d \ln m_{Z}^{2}}{d \ln |\mu|^{2}}\right| \simeq 2 \frac{|\mu|^{2}}{m_{Z}^{2}}, \Delta_{\delta m_{H_{u}}^{2}} \equiv\left|\frac{d \ln m_{Z}^{2}}{d \ln \delta m_{H_{u}}^{2}}\right| \simeq 2\left|\frac{\delta m_{H_{u}}^{2}}{m_{Z}^{2}}\right|,
$$

where we treat $\delta m_{H_{u}}^{2}$ as a fundamental parameter that is independent of other parameters. We also assume that the $\mu$-parameter is a fundamental parameter since it is an unique supersymmetry preserving parameter. Then, $\Delta_{\mu}$ increases as $\mu$-parameter increases quadratically. We see that $\Delta_{\mu} \sim \Delta_{\delta m_{H_{u}}^{2}}$ since the minimization condition eq. (3.4) requires $|\mu|^{2} \simeq \delta m_{H_{u}}^{2}$.

We assume that some suitable mechanism fixes $\alpha$ to satisfy $M_{\text {mir }}=M_{\text {SUSY }}$. If we consider the KKLT-like setups behind the mirage mediation, the value of $\alpha$ may be fixed by rational parameters and it is irrelevant to this tuning argument. Thus the size of the modulus mediation $M_{0}$ is important for the argument.

In eq. (3.6), $M_{\text {mir }}$ implicitly depends on $M_{0}$ through eq. (2.8). When we assume that the Kähler potential of the moduli is

$$
K_{\text {moduli }}=-3 \ln (T+\bar{T}),
$$

and only the modulus $T$ gets non-zero F-term vacuum expectation value, the size of the anomaly mediation $F^{C} / C$ relates to $M_{0}$ and $\alpha$ as

$$
\begin{aligned}
\frac{F^{C}}{C} & \equiv \alpha M_{0} \ln \left(\frac{M_{p}}{m_{3 / 2}}\right) \\
& =m_{3 / 2}+\frac{1}{3} K_{T} F^{T}=m_{3 / 2}-M_{0}
\end{aligned}
$$

where $K_{T} \equiv \partial K / \partial T$. Using above relations, we obtain

$$
\frac{d M_{\mathrm{mir}}}{d M_{0}}=\frac{\alpha}{2} \frac{m_{3 / 2}}{m_{3 / 2}+\alpha M_{0}} \frac{M_{\mathrm{mir}}}{M_{0}} \equiv c \frac{M_{\mathrm{mir}}}{M_{0}} .
$$

Note that $c \sim 1$ because $m_{3 / 2} \gg M_{0}$ and $\alpha \sim 2$ when $M_{\text {mir }} \sim M_{0}$.

Therefore the sensitivity to $M_{0}$ is

$$
\left|\frac{d \ln m_{Z}^{2}}{d \ln M_{0}^{2}}\right| \simeq \frac{M_{0}}{m_{Z}^{2}}\left|\frac{d m_{H_{u}}^{2}}{d M_{0}}\right| \simeq \frac{M_{0}}{m_{Z}^{2}}\left|c \frac{\gamma_{H_{u}}}{4 \pi^{2}} M_{0}+\frac{d \Delta m_{H_{u}}^{2}}{d M_{0}}\right|,
$$

where we choose $Q=M_{\mathrm{SUSY}}=M_{\mathrm{mir}}=M_{0} / \sqrt{2}$. We see that the sensitivity is suppressed by the loop factor. In our numerical analysis, we evaluate the correction from the 1-loop Coleman-Weinberg potential $[41,42]$ induced by the third-generation quarks and squarks.

Figure 1 shows the degree of tuning of $M_{0}$ and the $\mu$-parameter. We see that $\Delta_{M_{0}} \lesssim$ 100,250 and 1000 when $M_{0} \lesssim 4.6,6.8$ and $14 \mathrm{TeV}$, respectively. $\Delta_{\mu} \leq 100$ when $|\mu| \lesssim$ $650 \mathrm{GeV}$. In the case $\mu \simeq 1.1 \mathrm{TeV}$ where the thermal relic density of the higgsino saturates 

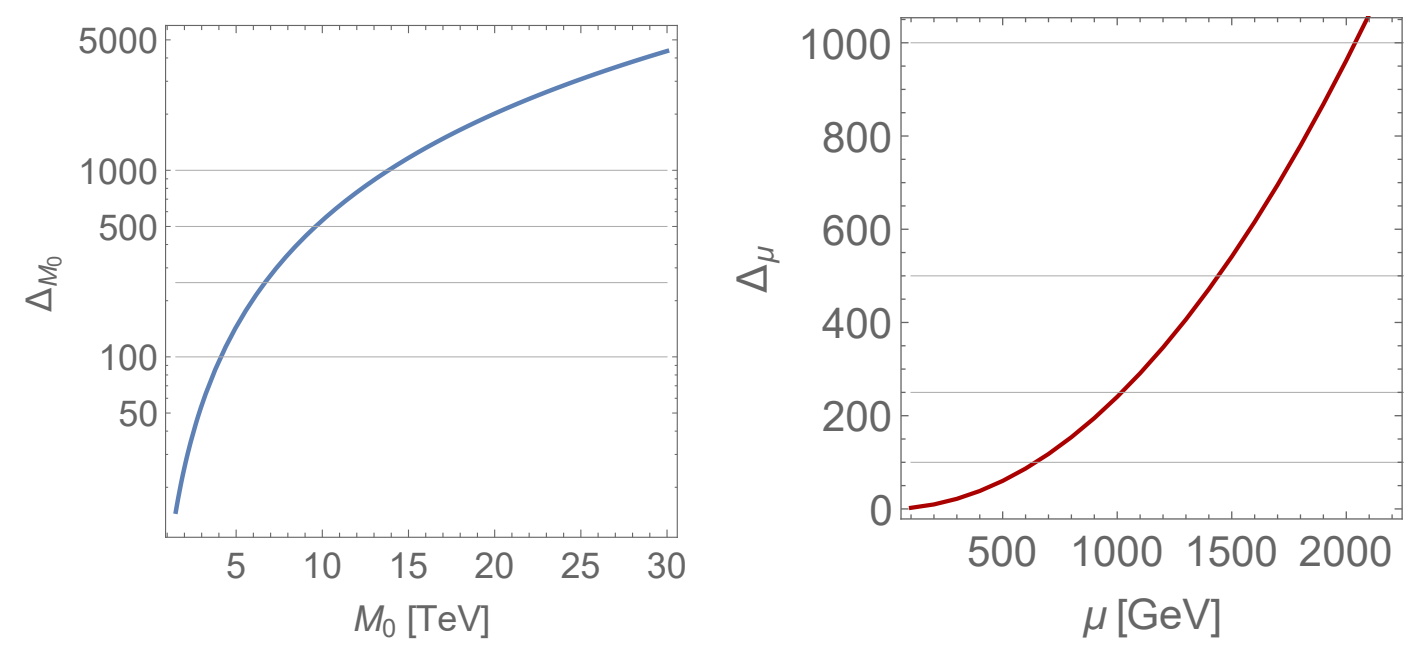

Figure 1. Degrees of tuning of $M_{0}$ (left) and $\mu$ (right). The gray lines correspond to $\Delta_{M_{0}, \mu}=$ 100, 250, 500, 1000 from bottom to top.

the observed value of DM, $\Delta_{\mu} \simeq 290$ and the degree of tuning is about $0.34 \%$. Since the $125 \mathrm{GeV}$ Higgs boson mass requires $M_{0} \gtrsim 6.0 \mathrm{TeV}$ as we will see later, the tuning of $M_{0}$ becomes severer than that of the $\mu$-parameter as long as $|\mu| \lesssim 1.1 \mathrm{TeV}$. In these regions, the electroweak symmetry breaking occurs with the parameter tuning about $0.3 \%$-level. This is much better than other scenarios with sparticles heavier than about $5 \mathrm{TeV}$.

\subsection{Direct LHC search}

In this setup, the extra Higgs bosons are expected to be lighter than the TeV scale and they could be discovered by the LHC experiments. The neutral Higgs bosons $H / A$ dominantly decay to a pair of bottom quarks with a branching fraction $\sim 80 \%$ and sub-dominantly decay to a pair of tau leptons with a branching fraction $\sim 10 \%$. When $\tan \beta$ is small and the bottom and the tau Yukawa couplings are suppressed, the Higgs bosons also decay to a pair of top quarks. The searches for $H, A \rightarrow \tau \tau$ however give the most stringent bound on their masses, since there are large amounts of backgrounds for the Higgs bosons decaying to quarks.

The experimental analyses constrain $\sigma(p p \rightarrow H / A) \times \operatorname{Br}(H / A \rightarrow \tau \tau)$, where the Higgs bosons are produced by the gluon fusion process or b-associated process [43]. In our analysis, we calculate the production cross section $\sigma(p p \rightarrow H / A)$ associated with bottom quark by using MadGraph-v2.5.4 with 5-flavor scheme [44]. We calculate the experimental limits based on the result of ref. [43] obtained by $36.1 \mathrm{fb}^{-1}$ data with the center of energy $\sqrt{s}=13 \mathrm{TeV}$.

The higgsino can be also detected by the collider experiments. There are two neutral and two charged components in the higgsino. Their masses tend to be mostly degenerate and mass differences are smaller than sub-GeV in our scenario. The mass differences come from the mixing with gauginos, and decrease as gaugino masses increase. The mass difference $\Delta m_{+} \equiv m_{\tilde{\chi}_{1}^{ \pm}}-m_{\tilde{\chi}_{1}^{0}}$ is smaller than about $\mathcal{O}(0.1 \mathrm{GeV})$ for $M_{0} \gtrsim 6.0 \mathrm{TeV}$ at the tree-level. $\Delta m_{+}$get smaller for larger $M_{0}$, but there are radiative corrections from SM 
gauge boson loops $[45,46]$. The radiative correction for the mass difference is given by

$$
\Delta m_{\mathrm{rad}}=\frac{\alpha_{2}}{8 \pi} m_{Z} s_{W}^{2} f\left(\frac{m_{Z}}{m_{\tilde{\chi}}}\right),
$$

where

$$
f(r)=2 r^{3} \ln r-2 r+\sqrt{r^{2}-4}\left(r^{2}+2\right) \ln \left(\frac{r^{2}-2-r \sqrt{r^{2}-4}}{2}\right) .
$$

Thus the size of the correction is $\Delta m_{\mathrm{rad}} \simeq \alpha_{2} m_{Z} s_{W}^{2} / 2 \sim 350 \mathrm{MeV}$ and would dominate the mass difference. The values of $\Delta m_{+}$including the loop effect in eq. (3.15) are shown in table 1. The LEP experiment gives the most stringent bound on such degenerate higgsinos [47]: the higgsino has to be heavier than about $90 \mathrm{GeV}$.

On the other hand, the expected signals are so weak that these are buried under the backgrounds in hadron collider experiments and the mass limits could not be severer than the one at the LEP. Recently, the higgsino search exploiting disappearing tracks are proposed in refs. [48, 49]. When the mass difference $\Delta m_{+} \equiv m_{\tilde{\chi}_{1}^{ \pm}}-m_{\tilde{\chi}_{1}^{0}}$ is smaller than about $0.6 \mathrm{GeV}$, the chargino dominantly decays to $e \nu_{e}, \mu \nu_{\mu}$ and $\pi^{+}$and the lightest neutralino. The partial decay widths are given by $[46,50,51]$

$$
\begin{aligned}
\Gamma\left(\tilde{\chi}_{1}^{+} \rightarrow \tilde{\chi}_{1}^{0} l \nu_{l}\right) & =\frac{2 G_{F}^{2}}{15 \pi^{3}}\left(\Delta m_{+}\right)^{5} \sqrt{1-x_{l}^{2}} \mathcal{P}\left(x_{l}\right), \quad(l=e, \mu), \\
\Gamma\left(\tilde{\chi}_{1}^{+} \rightarrow \tilde{\chi}_{1}^{0} \pi^{+}\right) & =\frac{2 G_{F}^{2} f_{\pi}^{2} \cos ^{2} \theta_{C}}{\pi}\left(\Delta m_{+}\right)^{3}\left(1-\frac{x_{\pi}^{2}}{2}\right) \sqrt{1-x_{\pi}},
\end{aligned}
$$

where $x_{l, \pi}=m_{l, \pi} / \Delta m_{+}, G_{F}$ is the Fermi constant, $f_{\pi} \sim 91.9 \mathrm{MeV}$ is the pion decay constant, $\theta_{C}$ is the Cabbibo angle and

$$
\mathcal{P}(x)=1-\frac{9}{2} x^{2}-4 x^{4}+\frac{15 x^{4}}{2 \sqrt{1-x^{2}}} \tanh ^{-1} \sqrt{1-x^{2}} .
$$

The decay length of the chargino could be longer than $\mathcal{O}(0.1 \mathrm{~cm})$ in our setup, so that the future hadron collider experiments would give stronger bounds than the LEP experiment. The decay lengths calculated from the above three decay modes are shown in table 1 . The expected exclusion limits at the HL-LHC and the 33-TeV hadron collider are shown in the next section, referring the result shown in ref. [49]. We refer the most optimistic cases in the future collider experiments, where there is no background events and the location of the second layer of a pixel detector is at a radius $3 \mathrm{~cm}$ in the $33-\mathrm{TeV}$ collider.

\subsection{Precision Higgs coupling measurement}

The light extra Higgs bosons change the couplings between the 125-GeV Higgs boson and SM particles; especially, couplings with bottom quark and tau lepton can be largely deviated from the SM prediction [52-56]. There are recent studies about the precision measurements of the Higgs couplings [57-59]. Now we define a ratio of a coupling in the MSSM to the one in the SM,

$$
\kappa_{f} \equiv g_{h \bar{f} f}^{\mathrm{MSSM}} / g_{h \bar{f} f}^{\mathrm{SM}},(f=b, \tau),
$$


where $g_{h \bar{f} f}^{\mathrm{I}}(I=\mathrm{MSSM}, \mathrm{SM})$ is the coupling between the $125-\mathrm{GeV}$ Higgs $(h)$ and the SM fermions $(f)$ :

$$
-\mathcal{L}_{h \bar{f} f}^{I}=g_{h \bar{f} f}^{I} h \bar{f} f
$$

$\kappa_{f}$ are written as

$$
\kappa_{f}=-\left(\frac{\sin \alpha_{h}}{\cos \beta}\right) \frac{1-\Delta_{f} \cot \alpha_{h} \cot \beta}{1+\Delta_{f}},
$$

where $\alpha_{h}$ is a mixing angle between two CP-even Higgs bosons. The first factor comes from purely Higgs boson mixing, and then it also exists in the type-II two Higgs doublet model. The factor, $\Delta_{f}$, comes from radiative corrections induced by sparticles. $\Delta_{b}, \Delta_{\tau}$ are approximately given by

$$
\begin{aligned}
\Delta_{b} & \simeq\left[\frac{2 \alpha_{s}}{3 \pi} M_{3} \mu I\left(m_{\tilde{b}_{1}}^{2}, m_{\tilde{b}_{2}}^{2}, M_{3}^{2}\right)+\frac{y_{t}^{2}}{16 \pi^{2}} \mu A_{t} I\left(m_{\tilde{t}_{1}}^{2}, m_{\tilde{t}_{2}}^{2}, \mu^{2}\right)\right] \tan \beta, \\
\Delta_{\tau} & \simeq-\frac{3 \alpha_{2}}{8 \pi} M_{2} \mu I\left(m_{\tilde{\tau}_{L}}^{2}, M_{2}^{2}, \mu^{2}\right) \times \tan \beta,
\end{aligned}
$$

where $I(a, b, c)$ is defined as

$$
I(a, b, c)=-\frac{a b \ln a / b+b c \ln b / c+c a \ln c / a}{(a-b)(b-c)(c-a)} .
$$

Note that these corrections are enhanced by $\tan \beta$. We calculate $\kappa_{f}$, using FeynHiggs 2.12 .2 which includes the re-summation of the $\Delta_{b}$ corrections [60] and the two-loop SUSY QCD corrections [61]. We have checked that the other couplings of the SM-like Higgs boson are very close to the SM value. The current accuracy of the Higgs coupling measurements at the LHC is $\mathcal{O}(10 \%)[62,63]$. The future sensitivity may reach a few $\%$ in the HL-LHC and the measurement may be more accurate than $1 \%$ in the future lepton collider experiments such as ILC and TLEP $[64,65]$. The HL-LHC will be able to measure $\kappa_{\tau}$ more precisely, while $\kappa_{b}$ can be determined more precisely at the lepton collider experiments.

\subsection{Flavor physics}

In our model, the flavor violating couplings involving sparticles are only given by the CKM matrix. Then, we can evade the strong bounds from flavor physics. It is known that the stringent bound on this kind of model comes from the rare $B$ meson decays: $B \rightarrow X_{s} \gamma$ [66$68]$ and $B_{s} \rightarrow \mu^{+} \mu^{-}[69,70]$. If there is only one extra Higgs doublet that couples to the right-handed down-type quarks as in the type-II $2 \mathrm{HDM}$, there is an one-loop correction involving charged Higgs which does not depend on $\tan \beta$.

In our supersymmetric model, there is also a superpartner of Higgs field, namely higgsino, below $1 \mathrm{TeV}$. There may be a cancellation between the charged Higgs loop and the higgsino loop [71].

We calculate $\operatorname{Br}\left(B_{s} \rightarrow X_{s} \gamma\right)$ and $\operatorname{Br}\left(B_{s} \rightarrow \mu^{+} \mu^{-}\right)$by using micrOmegas-4.3.2 [72-76]. We adopt the experimental values as follows: $\operatorname{Br}(b \rightarrow s \gamma)_{\exp }=(3.32 \pm 0.16) \times 10^{-4}$ for $E_{\gamma}>1.6 \mathrm{GeV}[77]$ and $\operatorname{Br}\left(B_{s} \rightarrow \mu^{+} \mu^{-}\right)_{\exp }=(3.00 \pm 0.55) \times 10^{-9}$ [70, 78, 79]. The SM predictions we use are $\operatorname{Br}(b \rightarrow s \gamma)_{\mathrm{SM}}=(3.36 \pm 0.23) \times 10^{-4}$ for $E_{\gamma}>1.6 \mathrm{GeV}$ [67] and $\operatorname{Br}\left(B_{s} \rightarrow \mu^{+} \mu^{-}\right)_{\mathrm{SM}}=(3.60 \pm 0.18) \times 10^{-9}[70]$. 


\subsection{DM physics}

The neutral component of the higgsino is the LSP and a good candidate for the dark matter in our scenario. ${ }^{3}$ There are several ways to produce the higgsino in the early universe.

If the mirage mediation is realized by the KKLT-like setup and the moduli and the gravitino masses are below $\mathrm{PeV}$-scale, late-time decays of these particles produce the higgsino. However, it is known that the higgsino dark matter produced in such a way tends to overclose the universe [81]. Hence, the production of the higgsino should be suppressed, or the produced higgsino should be diluted by e.g. thermal inflation [82, 83]. If the SUSY breaking scale is enough heavy $M_{0} \gtrsim \mathcal{O}(10) \mathrm{TeV}$ and the higgsino mass is enough light $m_{\chi} \lesssim \mathcal{O}(100) \mathrm{GeV}$ that the higgsinos produced by the late-time decays annihilate enough efficiently, the overclose problem could be circumvented and the relic density could be explained by the higgsino produced by the non-thermal way [84]. Otherwise, the model should be extended to have another light supersymmetric dark matter, such as axino $[85,86]$. In this case, the dark matter is no longer the higgsino, while the dark matter could be the higgsino dark matter in the other cases.

It would be possible that the relic density of the higgsino is explained by the usual thermal freeze-out mechanism, when the higgsino mass is about $1.1 \mathrm{TeV}$ [45, 87]. This occurs if the gravitino and moduli fields decay earlier than the higgsino freezing-out, although the decay widths of the gravitino and the moduli should be larger than the ones naively expected from the KKLT-like setups. Another possibility is that the mass spectrum of the mirage mediation is realized by some other mechanisms than the KKLT-like setup. This scenario looks interesting from the bottom-up point of view.

In figure 2, we consider the case that the higgsino dark matter is only thermally produced and saturates the observed relic density: $\Omega_{\mathrm{DM}} h^{2}=0.1188 \pm 0.001$ [88]. In other case, we study the constraints from the direct and indirect detections of the dark matter, assuming that the observed relic density is fully occupied by the higgsino dark matter. We calculate the thermal relic density of the higgsino, spin-independent cross section and the annihilation cross section by using micrOmegas-4.3.2 [72-76].

Note that the discussion about the DM in this subsection would become irrelevant, if the observed DM relic density is explained by some particle(s) other than the higgsino. If the higgsino does not dominate the dark matter density, the limits from the DM observations are relaxed by the rescaling factor, $\xi \equiv \Omega_{\chi} / \Omega_{\mathrm{DM}}$, where $\Omega_{\chi}$ is the higgsino density and $\Omega_{\mathrm{DM}}$ is the total DM density. The cross section for the direct detection should be rescaled by the factor $\xi$ and the cross section for the indirect detection, which observes the cosmic rays originated from the annihilation of the higgsinos, should be rescaled by $\xi^{2}$, when our predictions are compared with the experimental results.

There are studies about the DM in the mirage mediation and other similar setups [16, 89, 90]. In the previous works, the size of modulus mediation is below sub-TeV, so that the $125-\mathrm{GeV}$ Higgs boson mass can not be realized. Furthermore, the constraints from the direct detections now become very strong, so that the gaugino masses should be heavier than sub- $\mathrm{TeV}$ as far as the higgsino dominates the DM relic density. This situation is

\footnotetext{
${ }^{3}$ See for a review of the supersymmetric dark matter, e.g. [80].
} 
similar to the one in ref. [91], where the phenomenology of the higgsino DM is studied in the Non-Universal Gaugino Mass scenario. The most important difference with the previous work in ref. [91] is that the sparticle masses are much heavier in our scenario, because the size of A-term is fixed by the mediation mechanism at relatively small values and the heavy top squark is required to explain the Higgs boson mass around $125 \mathrm{GeV}$. Moreover, there are relatively light exotic Higgs bosons.

The direct-detection experiments constrain a cross section of DM-nucleon scattering; especially, the spin-independent cross section gives stringent bounds on the parameter space of the MSSM. The spin-independent cross section for the higgsino DM is approximately given by,

$$
\begin{aligned}
\sigma_{N}^{\mathrm{SI}} \simeq & \frac{g^{2}}{4 \pi} \frac{m_{N}^{4}}{m_{h}^{4} m_{W}^{2}}\left(1+\frac{m_{N}}{m_{\chi}}\right)^{-2} \\
& \times\left[\left(\sum_{q=u, d, s} f_{q}+\frac{2}{9} f_{T G}\right) \lambda_{h \chi \chi}-\frac{m_{h}^{2}}{m_{H}^{2}}\left(\sum_{q=u, d, s} f_{T_{q}} t_{q}+\frac{2}{27} f_{T G} \sum_{q=c, b, t} t_{q}\right) \lambda_{H \chi \chi}\right]^{2} \\
= & \frac{g^{4}}{16 \pi} \frac{m_{N}^{4}}{m_{h}^{4}}\left(1+\frac{m_{N}}{m_{\chi}}\right)^{-2}\left(\frac{1}{M_{2}-|\mu|}+\frac{t_{W}^{2}}{M_{1}-|\mu|}\right)^{2} \\
& \times\left[\left(\sum_{q=u, d, s} f_{q}+\frac{2}{9} f_{T G}\right)\left(1 \pm s_{2 \beta}\right) \pm \frac{m_{h}^{2}}{m_{H}^{2}}\left|c_{2 \beta}\right|\left(\sum_{q=u, d, s} f_{T_{q}} t_{q}+\frac{2}{27} f_{T G} \sum_{q=c, b, t} t_{q}\right)\right]^{2},
\end{aligned}
$$

where $m_{N}$ is the nucleon mass, $m_{N} f_{T_{q}}^{N}=\left\langle N\left|m_{q} \bar{q} q\right| N\right\rangle$ and $f_{T G}=1-\sum_{q=u, d, s} f_{T_{q}} \cdot t_{q}=$ $-\cot \beta$ for $q=u, c, t$ and $t_{q}=\tan \beta$ for $q=d, s, b$. \pm corresponds to the relative sign of the gaugino masses and the $\mu$-parameter. $\lambda_{h \chi \chi}, \lambda_{H \chi \chi}$ are the higgsino-higgsino-Higgs boson couplings,

$$
\begin{aligned}
& \lambda_{h \chi \chi}=\frac{g}{2}\left(1+\operatorname{sgn}\left(\mu M_{1,2}\right) s_{2 \beta}\right) c_{W} m_{Z}\left(\frac{1}{M_{2}-|\mu|}+\frac{t_{W}^{2}}{M_{1}-|\mu|}\right), \\
& \lambda_{H \chi \chi}=\frac{g}{2} \operatorname{sgn}\left(\mu M_{1,2}\right) c_{2 \beta} c_{W} m_{Z}\left(\frac{1}{M_{2}-|\mu|}+\frac{t_{W}^{2}}{M_{1}-|\mu|}\right),
\end{aligned}
$$

where $c_{W}, t_{W}, s_{2 \beta}$ and $c_{2 \beta}$ are short for $\cos \theta_{W}, \tan \theta_{W}, \sin 2 \beta$ and $\cos 2 \beta$, respectively. We replaced $\alpha_{h}$ to $\beta$ by taking the decoupling limit $m_{A} \gg m_{h}$ and drop all contributions from the sparticles.

The higgsino-higgsino-Higgs boson couplings are originated from the higgsino-gauginoHiggs boson couplings in the gauge-basis. This means that the couplings are suppressed by the gaugino masses as can be read from eq. (3.28). The heavy Higgs boson contribution becomes constructive (destructive) when $\operatorname{sgn}\left(\mu M_{1,2}\right)=+1(-1)$. Besides, the light Higgs boson contribution is proportional to $1+\operatorname{sgn}\left(\mu M_{1,2}\right) s_{2 \beta}$. Thus the spin-independent becomes significantly large when $\operatorname{sgn}\left(\mu M_{1,2}\right)=+1$.

The XENON1T experiment [92, 93] and the PandaX-II experiment [94] give the most severe bounds on the spin-independent cross section. The current limit is about $10^{-10} \mathrm{pb}$ for $m_{\chi} \sim 100 \mathrm{GeV}$ and $10^{-9} \mathrm{pb}$ for $m_{\chi} \sim 1100 \mathrm{GeV}$. There are future experiments such as 
LZ experiment [95], XENON-nT experiment and so on. These experiments will probe wide parameter space as long as the spin-independent cross section times the rescaling factor $\xi \sigma_{\mathrm{SI}}$ is on the so-called neutrino floor [96]. If $\xi \sigma_{\mathrm{SI}}$ is below the neutrino floor, the signals of the dark matter are buried under the neutrino background.

If the dark matter is dominated by the higgsino, we will observe cosmic rays originated from the higgsino annihilation. In most parameter region, a higgsino pair annihilates to a pair of W-bosons and of Z-bosons through the t-channel higgsino exchange except for the case with $m_{A} \simeq 2 m_{\chi}$. An important fact is that these processes are independent of other sparticles masses, because these are mediated by the higgsino itself. Thus the indirect detections constrain the higgsino mass itself. If $m_{A} \simeq 2 m_{\chi}$ is satisfied, a higgsino pair annihilate into a pair of bottom quarks (sub-dominantly tau leptons) through the s-channel CP-odd Higgs boson exchange. Although this contribution potentially becomes sizable, the higgsino-higgsino-Higgs boson coupling is suppressed by the gaugino masses as in the spin-independent cross section, so that these processes can not dominate the total annihilation process unless the Higgs boson mass is at the resonance region precisely. We could not observe such enhancement of the annihilation cross section in our numerical analysis in next section.

The current limit on the annihilation cross section comes from the AMS-02 experiment [97] which detects anti-protons. Analyses in refs. [98, 99] show the upper limits on the cross section, and the higgsino mass lighter than about $500 \mathrm{GeV}$ has been already excluded [91] if the higgsino saturates the dark matter density. The future experiments, such as the CTA experiment [100], would reach the cross section, $\sim 1.0 \times 10^{-26} \mathrm{~cm}^{3} / \mathrm{s}$, although there is a large uncertainty due to the unknown profiles of the dark matter.

\section{Numerical analysis}

Figure 2 shows the observables in the case that the thermal relic density of the higgsino saturates the observed value: $\Omega_{\chi} h^{2}=0.1188 \pm 0.001$. In this case, the higgsino mass is about $1.1 \mathrm{TeV}$. In figure 2, the sign of the $\mu$-parameter is the same (opposite) as the gaugino masses in the right (left) panel. The size of the modulus mediation $M_{0}$ is chosen to realize $m_{h}=125.09 \pm 0.01 \mathrm{GeV}$ and is shown by the background colors. The gray dashed lines correspond to $\Delta_{M_{0}}=400,300,250$ from bottom to top, where $M_{0}$ is estimated as $M_{0} \simeq$ 6.0, 7.4, 8.6 TeV, respectively. $\Delta_{M_{0}} \leq 300$ is satisfied above the line where $\tan \beta \gtrsim 32$ (38) when the sign of the $\mu$-parameter is opposite (same) to the gaugino masses. The degree of the tuning of $M_{0}$ is about $0.33 \%$ in this region. Note that $\mu \simeq 1.1 \mathrm{TeV}$ means that the $\mu$-parameter should be tuned about $0.3 \%$-level and the required tunings are comparable.

The red region is excluded by the LHC direct search for the extra neutral Higgs bosons decaying to a pair of tau leptons. The red lines correspond to $\sigma_{\text {prod }}(p p \rightarrow H / A) \times \operatorname{Br}(H / A \rightarrow$ $\tau \tau)=10^{-1}, 10^{-3} \mathrm{pb}$ from left to right. The exclusion limit for $m_{A}$ is tighter for larger $\tan \beta$ because of the larger production cross section and branching fraction to a pair of tau leptons. The current limit is $m_{A} \gtrsim 1.4 \mathrm{TeV}$ for $\tan \beta=50$ while there is no limits on $m_{A}$ for $\tan \beta \lesssim 15$. Note that the limits are not so changed, even if the higgsino mass is different. 

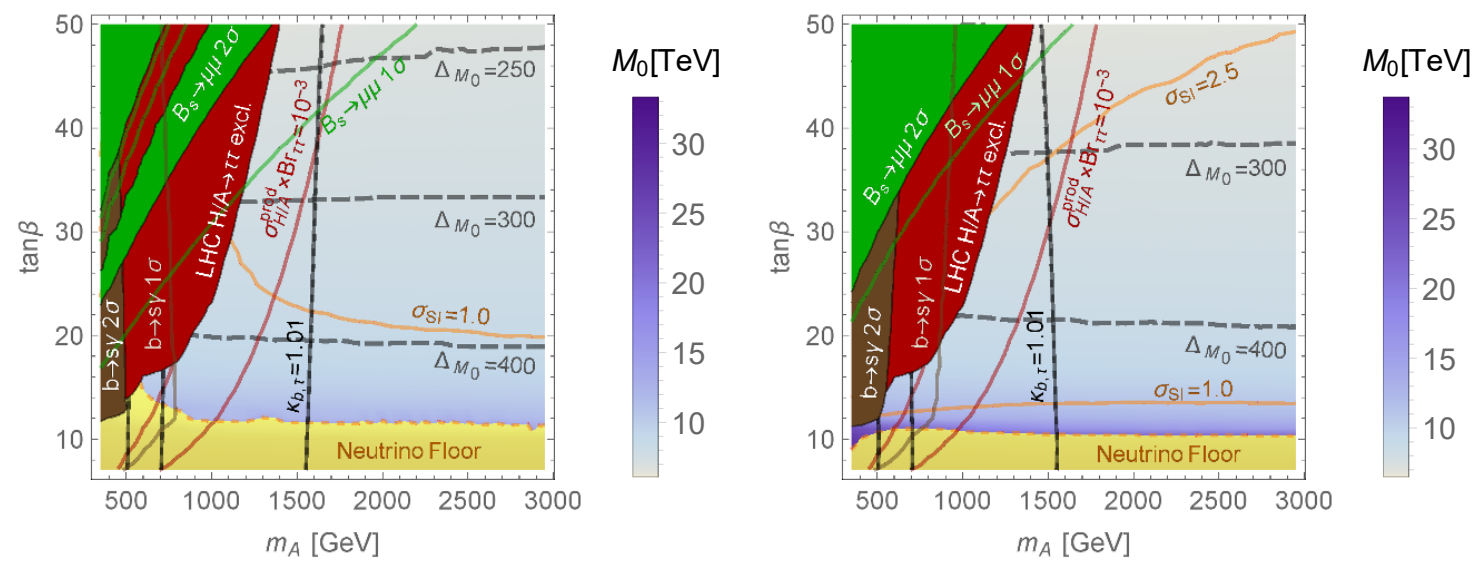

Figure 2. Values of the observables in the case with $\Omega_{\chi} h^{2}=0.1188 . \quad \operatorname{sgn}\left(\mu M_{1,2}\right)=-1(+1)$ in left (right) panel. The color of the background denotes the size of $M_{0}$. The dashed gray lines correspond to $\Delta_{M_{0}}=400,300,250$ from bottom to top. The green and brown regions are excluded by $B_{s} \rightarrow \mu^{+} \mu^{-}$and $b \rightarrow s \gamma$ at the $2 \sigma$ level. The 1- $\sigma$ exclusion limits are also depicted by the green and brown lines. The red region is excluded by the LHC direct search and the red lines correspond to $\sigma_{\text {prod }}(p p \rightarrow H / A) \times \operatorname{Br}(H / A \rightarrow \tau \tau)=10^{-1}, 10^{-3}[\mathrm{pb}]$ from left to right. The yellow region is below the neutrino floor of the DM direct detection. The spin-independent direct-detection cross sections are $\sigma_{S I}=1.0 \times 10^{-11}[\mathrm{pb}]$ and $2.5 \times 10^{-11}[\mathrm{pb}]$ on the yellow lines. The black solid (dashed) lines show $\kappa_{b, \tau}=1.10,1.05,1.01$ from left to right.

$\operatorname{Br}(b \rightarrow s \gamma)$ and $\operatorname{Br}\left(B_{s} \rightarrow \mu^{+} \mu^{-}\right)$are deviated from the central values at $2 \sigma$-level, in the brown and green regions, respectively. The uncertainties are calculated by combining the uncertainties of the SM prediction and the experimental results. The brown and green lines correspond to $1 \sigma$ deviation from the SM prediction. The measurement of $\operatorname{Br}(b \rightarrow s \gamma)$ excludes the light charged Higgs boson region even $\tan \beta$ is small. The measurement of $\operatorname{Br}\left(B_{s} \rightarrow \mu^{+} \mu^{-}\right)$excludes the light charged Higgs boson and heavy higgsino region as far as $\tan \beta$ is large, and the deviation reaches the $1 \sigma$-level from the current central value even if the extra Higgs bosons are so heavy that $\sigma_{H / A}^{\text {prod }} \times \operatorname{Br}(H / A \rightarrow \tau \tau)<10^{-3}$ pb is predicted.

The black solid (dashed) lines show $\kappa_{b, \tau}=1.10,1.05,1.01$ from left to right. Note that $\kappa_{b} \simeq \kappa_{\tau}$ is predicted since the deviations are mostly determined by the mixing between the Higgs bosons. The deviations from the SM predictions are more than $1 \%$ when the extra Higgs bosons are lighter than $1.5 \mathrm{TeV}$.

The yellow region shows that the spin-independent cross section is below the neutrino floor. That means that the yellow region is very difficult to be probed by the direct detections even if the dark matter is saturated by the higgsino. The yellow lines show the spin-independent cross section in the unit of $\times 10^{-11} \mathrm{pb}$. We see that the contribution from the heavy Higgs boson exchange reduces (enhances) the cross section when $\operatorname{sgn}\left(\mu M_{1,2}\right)=$ $-1(+1)$.

Figure 3 shows the results on $\mu-m_{A}$ planes with the fixed $\tan \beta$. The meanings of the red, brown, green and yellow regions and lines are the same as in figure 2. The gray region is excluded by the chargino search at the LEP experiment. The purple region is excluded by the AMS-02 experiment if the higgsino saturates the dark matter relic density. The 

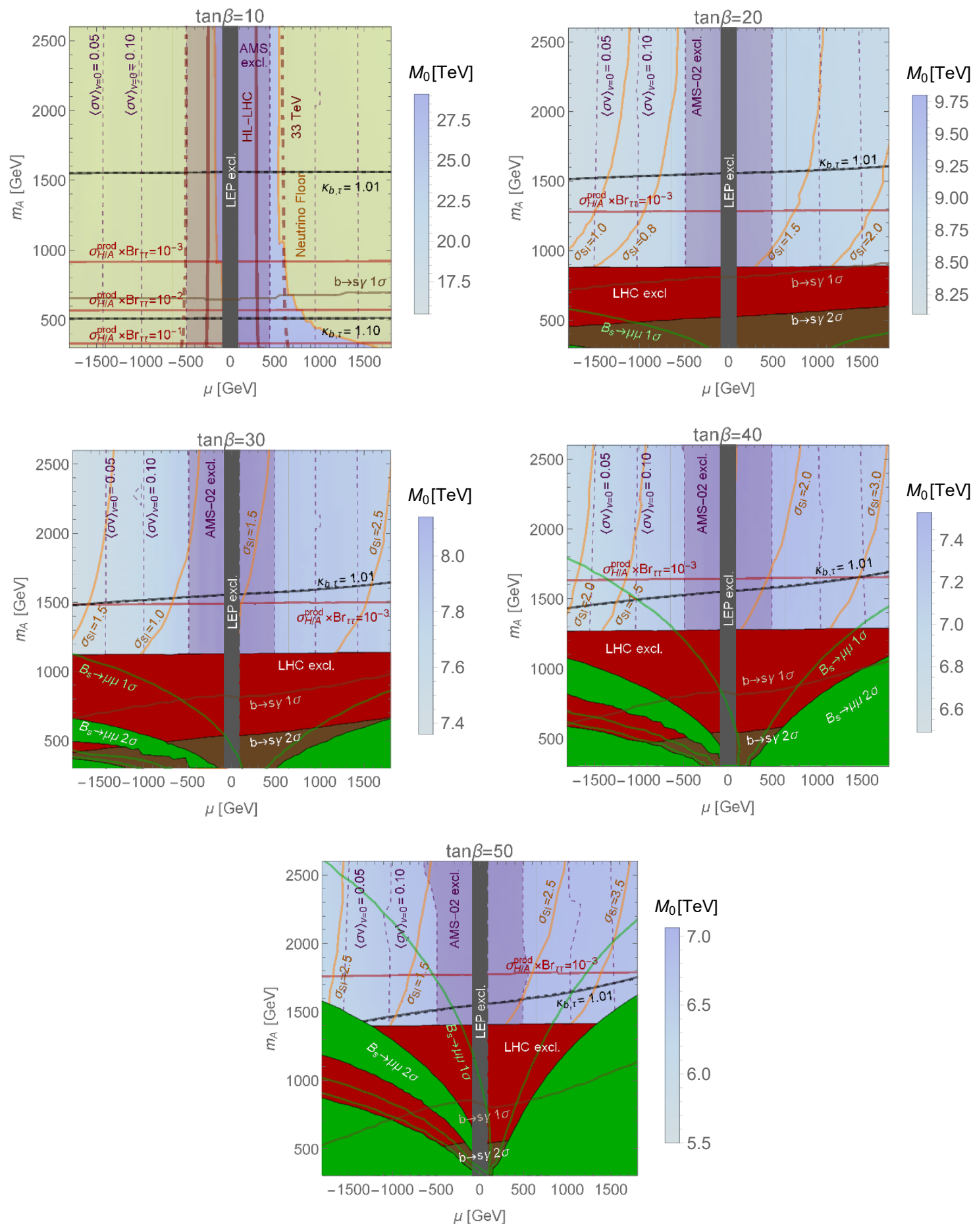

Figure 3. Values of the observables in the case $\tan \beta=10,20,30,40,50$. The meaning of the each line is the same as in figure 2. The gray and purple regions are excluded by the LEP experiment and the AMS-02 experiment. The purple dashed lines show the annihilation cross section in the unit of $\times 10^{-25} \mathrm{~cm}^{3} / \mathrm{s}$. 
purple dashed lines show the annihilation cross section in the zero velocity limit in the unit of $\times 10^{-25} \mathrm{~cm}^{3} / \mathrm{s}$.

The expected upper bounds on $\mu$ from the long-lived higgsino search at the HL-LHC (the 33-TeV hadron collider) are described by thick (dashed) red lines in the panel with $\tan \beta=10$. Most of the region with $\Delta_{\mu} \leq 100$ could be covered by the long-lived particle search at the $33 \mathrm{TeV}$ hadron collider. This region can be covered by the indirect detections for the dark matter only if the higgsino dominates the dark matter density. Thus the long-lived search is crucial to test the region where $\Delta_{\mu} \leq 100$ in the TeV-scale mirage mediation. Unfortunately, the long-lived particle search could not probe our scenario for larger $\tan \beta$ because of the lighter gaugino masses.

When $M_{0} \lesssim 10 \mathrm{TeV}$ is satisfied, $m_{A}, \mu$ are expected to be below sub-TeV due to the vanishing modulus mediation for the Higgs soft masses. The current limit on the parameter space comes from the direct search for the extra Higgs bosons except large $\mu$ parameter region with $\tan \beta=50$, where the bound from $\operatorname{Br}\left(B_{s} \rightarrow \mu^{+} \mu^{-}\right)$becomes severe. Those limits are highly dependent on $\tan \beta$. Note that the $125-\mathrm{GeV}$ Higgs boson couplings to the bottom quarks and the tau leptons deviate from the SM value at 1\%-level when $m_{A} \lesssim 1.5 \mathrm{TeV}$.

The yellow lines show the spin-independent cross section. The cross section is under the neutrino floor at $\tan \beta=10$, while whole region in the figures will be covered by the future experiment if the higgsino is the dark matter. The cross section depends on the heavier Higgs boson mass significantly and it increases (decreases) as $m_{A}$ increases, when $\mu$ is same (opposite) sign as the gaugino masses as can be read from eq. (3.26).

The annihilation cross section at zero-velocity limit is above $1.0 \times 10^{-26} \mathrm{~cm}^{3} / \mathrm{s}$ at $|\mu| \lesssim$ $1.0 \mathrm{TeV}$, so that the future indirect detections could cover the region where the degree of tuning the $\mu$-parameter is $\mathcal{O}(0.1) \%$-level. The event rate of the annihilation reduces significantly as the abundance of the higgsino decreases because the event rate is suppressed by a square of the rescaled factor $\xi=\Omega_{\chi} / \Omega_{\mathrm{DM}}$.

\section{Conclusion}

In this paper, we have studied the $\mathrm{TeV}$-scale mirage mediation scenario that can explain the EW scale naturally without conflict with the current experimental results.

A specific feature of the mirage mediation is that the RG effects for the soft parameters given by the modulus mediation are compensated by the anomaly mediated contributions below the unification scale, and both contributions cancel out at the mirage scale. This cancellation also happens in the soft SUSY scalar masses as well as the gaugino masses, if the modulus mediation respects the condition eq. (2.7), namely the mirage condition. Exploiting this unification feature, $m_{H_{u}}^{2}$ and the $\mu$-parameter, which are relevant to the EW symmetry breaking, can be smaller than the other soft parameters when the modulus mediated contributions to the Higgs soft parameters are vanishing at the gauge-coupling unification scale $\left(M_{U}\right)^{4}$ and the mirage scale is near the $\mathrm{TeV}$ scale.

\footnotetext{
${ }^{4}$ We define $c_{H_{u, d}}$ at $M_{U}$ and require $c_{H_{u, d}}=0$ in eq. (2.10).
} 
The mirage condition leads small $m_{H_{u}}^{2}$, but it also predicts small top squark mixing. Then the top squark mass should be heavier than about $5 \mathrm{TeV}$ to realize the $125-\mathrm{GeV}$ Higgs. This fact indicates that all superpartners except the higgsino are also heavier than $5 \mathrm{TeV}$ in our scenario and consistent with the stringent bounds on masses of superpartners with color at the LHC.

The assignment eq. (2.10) leads light extra Higgs bosons, and then the effective theory below the SUSY scale is like the type-II two-Higgs double model (2HDM) accompanied with the higgsinos. The apparent difference from the $2 \mathrm{HDM}$ is the existence of the higgsinos. The higgsino could be the dark matter and can be detected by the dark matter experiments. The higgsino in our scenario will be searched by not only the lepton collider but also the hadron collider exploiting disappearing tracks if $\tan \beta \lesssim 10$. The light higgsino also influences physical observables such as $\operatorname{Br}\left(B_{s} \rightarrow \mu^{+} \mu^{-}\right), \operatorname{Br}(b \rightarrow s \gamma)$ and the decay of the $125-\mathrm{GeV}$ Higgs boson, so that these would be different from the predictions in the 2HDM. The differences are getting significant as $\tan \beta$ increases.

We emphasize that the $\mathrm{TeV}$-scale mirage mediation discussed in this paper still holds motivations of the low-scale supersymmetry. The EW scale can be explained by tuning the parameters at $0.3 \%$-level. The mirage unification features allows us to control the soft parameters at low energy and the parameters relevant to the EW symmetry breaking can remain below the $\mathrm{TeV}$-scale. In our scenario, the higgsino is a candidate for the dark matter. The extra Higgs bosons and the higgsinos are expected to be below TeV-scale, so that they can be explored by the current and upcoming experiments in both the direct and indirect ways.

\section{Acknowledgments}

The work of J. K. is supported by Grant-in-Aid for Research Fellow of Japan Society for the Promotion of Science No. 16J04215. The work of Y. O. is supported by Grant-in-Aid for Scientific research from the Ministry of Education, Science, Sports, and Culture (MEXT), Japan, No. 17H05404.

Open Access. This article is distributed under the terms of the Creative Commons Attribution License (CC-BY 4.0), which permits any use, distribution and reproduction in any medium, provided the original author(s) and source are credited.

\section{References}

[1] S.P. Martin, A supersymmetry primer, Adv. Ser. Direct. High Energy Phys. 21 (2010) 1 [Adv. Ser. Direct. High Energy Phys. 18 (1998) 1] [hep-ph/9709356] [INSPIRE].

[2] D.J.H. Chung et al., The soft supersymmetry breaking lagrangian: theory and applications, Phys. Rept. 407 (2005) 1 [hep-ph/0312378] [INSPIRE].

[3] ATLAS and CMS collaborations, Combined measurement of the Higgs boson mass in pp collisions at $\sqrt{s}=7$ and $8 \mathrm{TeV}$ with the ATLAS and CMS experiments, Phys. Rev. Lett. 114 (2015) 191803 [arXiv: 1503.07589] [INSPIRE]. 
[4] H. Abe, T. Kobayashi and Y. Omura, Relaxed fine-tuning in models with non-universal gaugino masses, Phys. Rev. D 76 (2007) 015002 [hep-ph/0703044] [INSPIRE].

[5] H. Abe, J. Kawamura and H. Otsuka, The Higgs boson mass in a natural MSSM with nonuniversal gaugino masses at the GUT scale, PTEP 2013 (2013) $013 \mathrm{~B} 02$ [arXiv: 1208.5328] [INSPIRE].

[6] H. Abe, J. Kawamura and Y. Omura, LHC phenomenology of natural MSSM with non-universal gaugino masses at the unification scale, JHEP 08 (2015) 089 [arXiv: 1505.03729] [INSPIRE].

[7] J. Kawamura and Y. Omura, Constraints on nonuniversal gaugino mass scenario using the latest LHC data, Phys. Rev. D 93 (2016) 055019 [arXiv:1601.03484] [INSPIRE].

[8] K. Choi, K.S. Jeong, T. Kobayashi and K.-i. Okumura, Little SUSY hierarchy in mixed modulus-anomaly mediation, Phys. Lett. B 633 (2006) 355 [hep-ph/0508029] [INSPIRE].

[9] K. Choi, K.S. Jeong, T. Kobayashi and K.-i. Okumura, TeV scale mirage mediation and natural little SUSY hierarchy, Phys. Rev. D 75 (2007) 095012 [hep-ph/0612258] [INSPIRE].

[10] R. Kitano and Y. Nomura, A solution to the supersymmetric fine-tuning problem within the MSSM, Phys. Lett. B 631 (2005) 58 [hep-ph/0509039] [INSPIRE].

[11] T. Kobayashi, D. Suematsu, K. Yamada and Y. Yamagishi, Nonuniversal soft scalar masses in superstring theories, Phys. Lett. B 348 (1995) 402 [hep-ph/9408322] [INSPIRE].

[12] A. Brignole, L.E. Ibáñez, C. Muñoz and C. Scheich, Some issues in soft SUSY breaking terms from dilaton/moduli sectors, Z. Phys. C 74 (1997) 157 [hep-ph/9508258] [INSPIRE].

[13] A. Brignole, L.E. Ibáñez and C. Muñoz, Soft supersymmetry breaking terms from supergravity and superstring models, Adv. Ser. Direct. High Energy Phys. 18 (1998) 125 [hep-ph/9707209] [INSPIRE].

[14] L. Randall and R. Sundrum, Out of this world supersymmetry breaking, Nucl. Phys. B 557 (1999) 79 [hep-th/9810155] [INSPIRE].

[15] G.F. Giudice, M.A. Luty, H. Murayama and R. Rattazzi, Gaugino mass without singlets, JHEP 12 (1998) 027 [hep-ph/9810442] [INSPIRE].

[16] K. Choi, K.Y. Lee, Y. Shimizu, Y.G. Kim and K.-i. Okumura, Neutralino dark matter in mirage mediation, JCAP 12 (2006) 017 [hep-ph/0609132] [INSPIRE].

[17] W.S. Cho, Y.G. Kim, K.Y. Lee, C.B. Park and Y. Shimizu, LHC signature of mirage mediation, JHEP 04 (2007) 054 [hep-ph/0703163] [INSPIRE].

[18] M. Nagai and K. Nakayama, Nonthermal dark matter in mirage mediation, Phys. Rev. D 76 (2007) 123501 [arXiv:0709.3918] [INSPIRE].

[19] M. Asano and T. Higaki, Natural supersymmetric spectrum in mirage mediation, Phys. Rev. D 86 (2012) 035020 [arXiv: 1204.0508] [INSPIRE].

[20] T. Kobayashi, H. Makino, K.-i. Okumura, T. Shimomura and T. Takahashi, TeV scale mirage mediation in NMSSM, JHEP 01 (2013) 081 [arXiv:1204.3561] [INSPIRE].

[21] H. Abe and J. Kawamura, The 126 GeV Higgs boson mass and naturalness in (deflected) mirage mediation, JHEP 07 (2014) 077 [arXiv: 1405.0779] [INSPIRE].

[22] K. Hagimoto, T. Kobayashi, H. Makino, K.-i. Okumura and T. Shimomura, Phenomenology of NMSSM in TeV scale mirage mediation, JHEP 02 (2016) 089 [arXiv:1509.05327] [INSPIRE]. 
[23] H. Baer, V. Barger, H. Serce and X. Tata, Natural generalized mirage mediation, Phys. Rev. D 94 (2016) 115017 [arXiv: 1610.06205] [InSPIRE].

[24] U. Ellwanger, C. Hugonie and A.M. Teixeira, The next-to-minimal supersymmetric standard model, Phys. Rept. 496 (2010) 1 [arXiv:0910.1785] [INSPIRE].

[25] K. Choi, A. Falkowski, H.P. Nilles, M. Olechowski and S. Pokorski, Stability of flux compactifications and the pattern of supersymmetry breaking, JHEP 11 (2004) 076 [hep-th/0411066] [INSPIRE].

[26] K. Choi, A. Falkowski, H.P. Nilles and M. Olechowski, Soft supersymmetry breaking in KKLT flux compactification, Nucl. Phys. B 718 (2005) 113 [hep-th/0503216] [InSPIRE].

[27] K. Choi, K.S. Jeong and K.-i. Okumura, Phenomenology of mixed modulus-anomaly mediation in fluxed string compactifications and brane models, JHEP 09 (2005) 039 [hep-ph/0504037] [INSPIRE].

[28] S. Kachru, R. Kallosh, A.D. Linde and S.P. Trivedi, De Sitter vacua in string theory, Phys. Rev. D 68 (2003) 046005 [hep-th/0301240] [INSPIRE].

[29] H. Abe, T. Higaki and T. Kobayashi, KKLT type models with moduli-mixing superpotential, Phys. Rev. D 73 (2006) 046005 [hep-th/0511160] [INSPIRE].

[30] H. Abe, T. Higaki, T. Kobayashi and Y. Omura, Moduli stabilization, F-term uplifting and soft supersymmetry breaking terms, Phys. Rev. D 75 (2007) 025019 [hep-th/0611024] [INSPIRE].

[31] A. Djouadi, J.-L. Kneur and G. Moultaka, SuSpect: a Fortran code for the supersymmetric and Higgs particle spectrum in the MSSM, Comput. Phys. Commun. 176 (2007) 426 [hep-ph/0211331] [INSPIRE].

[32] M. Muhlleitner, A. Djouadi and Y. Mambrini, SDECAY: a Fortran code for the decays of the supersymmetric particles in the MSSM, Comput. Phys. Commun. 168 (2005) 46 [hep-ph/0311167] [INSPIRE].

[33] A. Djouadi, M.M. Muhlleitner and M. Spira, Decays of supersymmetric particles: The Program SUSY-HIT (SUspect-SdecaY-HDECAY-InTerface), Acta Phys. Polon. B 38 (2007) 635 [hep-ph/0609292] [inSPIRE].

[34] S. Heinemeyer, W. Hollik and G. Weiglein, FeynHiggs: a program for the calculation of the masses of the neutral CP even Higgs bosons in the MSSM, Comput. Phys. Commun. 124 (2000) 76 [hep-ph/9812320] [INSPIRE].

[35] S. Heinemeyer, W. Hollik and G. Weiglein, The masses of the neutral CP-even Higgs bosons in the MSSM: accurate analysis at the two loop level, Eur. Phys. J. C 9 (1999) 343 [hep-ph/9812472] [INSPIRE].

[36] G. Degrassi, S. Heinemeyer, W. Hollik, P. Slavich and G. Weiglein, Towards high precision predictions for the MSSM Higgs sector, Eur. Phys. J. C 28 (2003) 133 [hep-ph/0212020] [INSPIRE].

[37] M. Frank, T. Hahn, S. Heinemeyer, W. Hollik, H. Rzehak and G. Weiglein, The Higgs boson masses and mixings of the complex MSSM in the Feynman-diagrammatic approach, JHEP 02 (2007) 047 [hep-ph/0611326] [INSPIRE].

[38] T. Hahn, S. Heinemeyer, W. Hollik, H. Rzehak and G. Weiglein, High-precision predictions for the light CP-even Higgs boson mass of the minimal supersymmetric standard model, Phys. Rev. Lett. 112 (2014) 141801 [arXiv:1312.4937] [INSPIRE]. 
[39] H. Bahl and W. Hollik, Precise prediction for the light MSSM Higgs boson mass combining effective field theory and fixed-order calculations, Eur. Phys. J. C 76 (2016) 499 [arXiv: 1608.01880] [INSPIRE].

[40] R. Barbieri and G.F. Giudice, Upper bounds on supersymmetric particle masses, Nucl. Phys. B 306 (1988) 63 [inSPIRE].

[41] S.R. Coleman and E.J. Weinberg, Radiative corrections as the origin of spontaneous symmetry breaking, Phys. Rev. D 7 (1973) 1888 [InSPIRE].

[42] S. Weinberg, Perturbative calculations of symmetry breaking, Phys. Rev. D 7 (1973) 2887 [INSPIRE].

[43] ATLAS collaboration, Search for additional heavy neutral Higgs and gauge bosons in the ditau final state produced in $36.1 \mathrm{fb}^{-1}$ of pp collisions at $\sqrt{\mathrm{s}}=13 \mathrm{TeV}$ with the ATLAS detector, ATLAS-CONF-2017-050 (2017).

[44] J. Alwall et al., The automated computation of tree-level and next-to-leading order differential cross sections and their matching to parton shower simulations, JHEP $\mathbf{0 7}$ (2014) 079 [arXiv : 1405.0301] [inSPIRE].

[45] M. Cirelli, N. Fornengo and A. Strumia, Minimal dark matter, Nucl. Phys. B 753 (2006) 178 [hep-ph/0512090] [INSPIRE].

[46] S.D. Thomas and J.D. Wells, Phenomenology of massive vectorlike doublet leptons, Phys. Rev. Lett. 81 (1998) 34 [hep-ph/9804359] [INSPIRE].

[47] ALEPH collaboration, A. Heister et al., Search for charginos nearly mass degenerate with the lightest neutralino in $e^{+} e^{-}$collisions at center-of-mass energies up to $209 \mathrm{GeV}$, Phys. Lett. B 533 (2002) 223 [hep-ex/0203020] [INSPIRE].

[48] R. Mahbubani, P. Schwaller and J. Zurita, Closing the window for compressed dark sectors with disappearing charged tracks, JHEP 06 (2017) 119 [Erratum ibid. 10 (2017) 061] [arXiv: 1703.05327] [INSPIRE].

[49] H. Fukuda, N. Nagata, H. Otono and S. Shirai, Higgsino dark matter or not: role of disappearing track searches at the LHC and future colliders, arXiv:1703.09675 [INSPIRE].

[50] C.H. Chen, M. Drees and J.F. Gunion, A nonstandard string/SUSY scenario and its phenomenological implications, Phys. Rev. D 55 (1997) 330 [Erratum ibid. D 60 (1999) 039901] [hep-ph/9607421] [INSPIRE].

[51] C.H. Chen, M. Drees and J.F. Gunion, Addendum/erratum for 'searching for invisible and almost invisible particles at $e^{+} e^{-}$colliders' [hep-ph/9512230] and 'a nonstandard string/SUSY scenario and its phenomenological implications' [hep-ph/9607421], hep-ph/9902309 [inSPIRE].

[52] L.J. Hall, R. Rattazzi and U. Sarid, The top quark mass in supersymmetric $\mathrm{SO}(10)$ unification, Phys. Rev. D 50 (1994) 7048 [hep-ph/9306309] [INSPIRE].

[53] R. Hempfling, Yukawa coupling unification with supersymmetric threshold corrections, Phys. Rev. D 49 (1994) 6168 [INSPIRE].

[54] M. Carena, M. Olechowski, S. Pokorski and C.E.M. Wagner, Electroweak symmetry breaking and bottom-top Yukawa unification, Nucl. Phys. B 426 (1994) 269 [hep-ph/9402253] [INSPIRE]. 
[55] M. Carena, S. Mrenna and C.E.M. Wagner, MSSM Higgs boson phenomenology at the Tevatron collider, Phys. Rev. D 60 (1999) 075010 [hep-ph/9808312] [INSPIRE].

[56] H. Eberl, K. Hidaka, S. Kraml, W. Majerotto and Y. Yamada, Improved SUSY QCD corrections to Higgs boson decays into quarks and squarks, Phys. Rev. D 62 (2000) 055006 [hep-ph/9912463] [INSPIRE].

[57] M. Endo, T. Moroi and M.M. Nojiri, Footprints of supersymmetry on Higgs decay, JHEP 04 (2015) 176 [arXiv: 1502.03959] [INSPIRE].

[58] K.J. Bae, H. Baer, N. Nagata and H. Serce, Prospects for Higgs coupling measurements in SUSY with radiatively-driven naturalness, Phys. Rev. D 92 (2015) 035006 [arXiv: 1505.03541] [INSPIRE].

[59] M. Kakizaki, S. Kanemura, M. Kikuchi, T. Matsui and H. Yokoya, Indirect reach of heavy MSSM Higgs bosons by precision measurements at future lepton colliders, Int. J. Mod. Phys. A 30 (2015) 1550192 [arXiv: 1505.03761] [INSPIRE].

[60] L. Hofer, U. Nierste and D. Scherer, Resummation of tan-beta-enhanced supersymmetric loop corrections beyond the decoupling limit, JHEP 10 (2009) 081 [arXiv:0907.5408] [INSPIRE].

[61] D. Noth and M. Spira, Higgs boson couplings to bottom quarks: two-loop supersymmetry-QCD corrections, Phys. Rev. Lett. 101 (2008) 181801 [arXiv:0808.0087] [INSPIRE].

[62] ATLAS collaboration, Measurements of the Higgs boson production and decay rates and coupling strengths using pp collision data at $\sqrt{s}=7$ and $8 \mathrm{TeV}$ in the ATLAS experiment, Eur. Phys. J. C 76 (2016) 6 [arXiv:1507.04548] [inSPIRE].

[63] CMS collaboration, Precise determination of the mass of the Higgs boson and tests of compatibility of its couplings with the standard model predictions using proton collisions at 7 and 8 TeV, Eur. Phys. J. C 75 (2015) 212 [arXiv:1412.8662] [INSPIRE].

[64] M.E. Peskin, Comparison of LHC and ILC capabilities for Higgs boson coupling measurements, arXiv:1207.2516 [INSPIRE].

[65] S. Dawson et al., Working group report: Higgs boson, arXiv:1310.8361 [INSPIRE].

[66] T. Hermann, M. Misiak and M. Steinhauser, $\bar{B} \rightarrow X_{s} \gamma$ in the two Higgs doublet model up to next-to-next-to-leading order in QCD, JHEP 11 (2012) 036 [arXiv:1208.2788] [INSPIRE].

[67] M. Misiak et al., Updated NNLO QCD predictions for the weak radiative B-meson decays, Phys. Rev. Lett. 114 (2015) 221801 [arXiv:1503.01789] [INSPIRE].

[68] M. Misiak and M. Steinhauser, Weak radiative decays of the $B$ meson and bounds on $M_{H^{ \pm}}$ in the Two-Higgs-Doublet Model, Eur. Phys. J. C 77 (2017) 201 [arXiv:1702.04571] [INSPIRE].

[69] W. Altmannshofer, M. Carena, N.R. Shah and F. Yu, Indirect probes of the MSSM after the Higgs discovery, JHEP 01 (2013) 160 [arXiv:1211.1976] [INSPIRE].

[70] W. Altmannshofer, C. Niehoff and D.M. Straub, $B_{s} \rightarrow \mu^{+} \mu^{-}$as current and future probe of new physics, JHEP 05 (2017) 076 [arXiv: 1702.05498] [INSPIRE].

[71] N. Chen, D. Feldman, Z. Liu and P. Nath, SUSY and Higgs signatures implied by cancellations in $b \rightarrow s \gamma$, Phys. Lett. B 685 (2010) 174 [arXiv:0911.0217] [INSPIRE]. 
[72] G. Bélanger, F. Boudjema, A. Pukhov and A. Semenov, MicrOMEGAs_3: a program for calculating dark matter observables, Comput. Phys. Commun. 185 (2014) 960 [arXiv:1305.0237] [INSPIRE].

[73] G. Bélanger, F. Boudjema, A. Pukhov and A. Semenov, MicrOMEGAs: version 1.3, Comput. Phys. Commun. 174 (2006) 577 [hep-ph/0405253] [INSPIRE].

[74] G. Bélanger, F. Boudjema, A. Pukhov and A. Semenov, MicrOMEGAs: a program for calculating the relic density in the MSSM, Comput. Phys. Commun. 149 (2002) 103 [hep-ph/0112278] [INSPIRE].

[75] G. Bélanger, F. Boudjema, A. Pukhov and A. Semenov, MicrOMEGAs4.1: two dark matter candidates, Comput. Phys. Commun. 192 (2015) 322 [arXiv:1407.6129] [InSPIRE].

[76] G. Bélanger, F. Boudjema, A. Pukhov and A. Semenov, Dark matter direct detection rate in a generic model with MicrOMEGAs 2.2, Comput. Phys. Commun. 180 (2009) 747 [arXiv:0803.2360] [INSPIRE].

[77] Y. Amhis et al., Averages of b-hadron, c-hadron and $\tau$-lepton properties as of summer 2016, arXiv: 1612.07233 [INSPIRE].

[78] LHCb and CMS collaborations, Observation of the rare $B_{s}^{0} \rightarrow \mu^{+} \mu^{-}$decay from the combined analysis of CMS and LHCb data, Nature 522 (2015) 68 [arXiv:1411.4413] [INSPIRE].

[79] LHCb collaboration, Measurement of the $B_{s}^{0} \rightarrow \mu^{+} \mu^{-}$branching fraction and effective lifetime and search for $B^{0} \rightarrow \mu^{+} \mu^{-}$decays, Phys. Rev. Lett. 118 (2017) 191801 [arXiv: 1703.05747] [INSPIRE].

[80] G. Jungman, M. Kamionkowski and K. Griest, Supersymmetric dark matter, Phys. Rept. 267 (1996) 195 [hep-ph/9506380] [INSPIRE].

[81] M. Endo, K. Hamaguchi and F. Takahashi, Moduli-induced gravitino problem, Phys. Rev. Lett. 96 (2006) 211301 [hep-ph/0602061] [INSPIRE].

[82] D.H. Lyth and E.D. Stewart, Cosmology with a TeV mass GUT Higgs, Phys. Rev. Lett. 75 (1995) 201 [hep-ph/9502417] [INSPIRE].

[83] D.H. Lyth and E.D. Stewart, Thermal inflation and the moduli problem, Phys. Rev. D 53 (1996) 1784 [hep-ph/9510204] [INSPIRE].

[84] L. Aparicio, M. Cicoli, B. Dutta, F. Muia and F. Quevedo, Light higgsino dark matter from non-thermal cosmology, JHEP 11 (2016) 038 [arXiv: 1607.00004] [INSPIRE].

[85] S. Nakamura, K.-i. Okumura and M. Yamaguchi, Axionic mirage mediation, Phys. Rev. D 77 (2008) 115027 [arXiv:0803.3725] [INSPIRE].

[86] K. Choi, K.S. Jeong, W.-I. Park and C.S. Shin, Thermal inflation and baryogenesis in heavy gravitino scenario, JCAP 11 (2009) 018 [arXiv:0908.2154] [INSPIRE].

[87] M. Cirelli, A. Strumia and M. Tamburini, Cosmology and astrophysics of minimal dark matter, Nucl. Phys. B 787 (2007) 152 [arXiv:0706.4071] [InSPIRE].

[88] Planck collaboration, P.A.R. Ade et al., Planck 2015 results. XIII. Cosmological parameters, Astron. Astrophys. 594 (2016) A13 [arXiv:1502.01589] [INSPIRE].

[89] H. Abe, Y.G. Kim, T. Kobayashi and Y. Shimizu, TeV scale partial mirage unification and neutralino dark matter, JHEP 09 (2007) 107 [arXiv:0706.4349] [INSPIRE]. 
[90] M. Holmes and B.D. Nelson, Dark matter prospects in deflected mirage mediation, JCAP 07 (2009) 019 [arXiv: 0905.0674] [INSPIRE].

[91] J. Kawamura and Y. Omura, Study of dark matter physics in non-universal gaugino mass scenario, JHEP 08 (2017) 072 [arXiv: 1703.10379] [INSPIRE].

[92] XENON collaboration, E. Aprile et al., First dark matter search results from the XENON1T experiment, Phys. Rev. Lett. 119 (2017) 181301 [arXiv:1705. 06655] [INSPIRE].

[93] XENON collaboration, E. Aprile et al., Physics reach of the XENON1T dark matter experiment, JCAP 04 (2016) 027 [arXiv: 1512.07501] [INSPIRE].

[94] PandaX-II collaboration, X. Cui et al., Dark matter results from 54-ton-day exposure of PandaX-II experiment, Phys. Rev. Lett. 119 (2017) 181302 [arXiv:1708.06917] [InSPIRE].

[95] LZ collaboration, D.S. Akerib et al., LUX-ZEPLIN (LZ) conceptual design report, arXiv: 1509.02910 [INSPIRE].

[96] J. Billard, L. Strigari and E. Figueroa-Feliciano, Implication of neutrino backgrounds on the reach of next generation dark matter direct detection experiments, Phys. Rev. D 89 (2014) 023524 [arXiv: 1307.5458] [INSPIRE].

[97] ANS collaboration, M. Aguilar et al., Antiproton flux, antiproton-to-proton flux ratio, and properties of elementary particle fluxes in primary cosmic rays measured with the Alpha Magnetic Spectrometer on the International Space Station, Phys. Rev. Lett. 117 (2016) 091103.

[98] A. Cuoco, M. Krämer and M. Korsmeier, Novel dark matter constraints from antiprotons in light of AMS-02, Phys. Rev. Lett. 118 (2017) 191102 [arXiv:1610.03071] [INSPIRE].

[99] M.-Y. Cui, Q. Yuan, Y.-L.S. Tsai and Y.-Z. Fan, Possible dark matter annihilation signal in the AMS-02 antiproton data, Phys. Rev. Lett. 118 (2017) 191101 [arXiv:1610.03840] [INSPIRE].

[100] CTA collaboration, J. Carr et al., Prospects for indirect dark matter searches with the Cherenkov Telescope Array (CTA), PoS(ICRC2015) 1203 [arXiv: 1508.06128] [INSPIRE]. 